\title{
Developmental features of DNA methylation in CpG islands of human gametes and preimplantation embryos
}

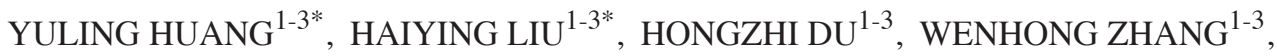 \\ XIANJING KANG ${ }^{1-3}$, YANG LUO ${ }^{1-3}$, XUELIANG ZHOU ${ }^{1-3}$ and LEI LI ${ }^{1-3}$ \\ ${ }^{1}$ Center for Reproductive Medicine, Third Affiliated Hospital of Guangzhou Medical University; ${ }^{2}$ Key Laboratory \\ for Reproductive Medicine of Guangdong Province; ${ }^{3}$ Key Laboratory for Major Obstetric Disease of Guangdong \\ Province, Key Laboratory of Reproduction and Genetics of Guangdong Higher Education Institutes, Third \\ Affiliated Hospital of Guangzhou Medical University, Guangzhou, Guangdong 510150, P.R. China
}

Received September 15, 2018; Accepted April 3, 2019

DOI: $10.3892 /$ etm.2019.7523

\begin{abstract}
The aim of current study was to apply the methylated DNA immunoprecipitation (MeDIP)-Chip method to investigate dynamic changes in $\mathrm{CpG}$ island methylation in human sperm, oocytes and various developmental stages of preimplantation embryos. Samples were divided into eight groups: 1, sperm ( $\mathrm{n}=30)$; 2, MII oocyte ( $\mathrm{n}=25)$; 3, two-pronuclear (2PN) period zygote $(\mathrm{n}=25) ; 4,4$-cell stage embryo $(\mathrm{n}=5) ; 5,8$-cell stage embryo $(\mathrm{n}=4) ; 6$, morula embryo $(\mathrm{n}=6)$; 7, blastular inner cell mass (ICM) group $(\mathrm{n}=5)$; 8, blastular trophoblastic cells (TE) $(n=5)$. DNA was extracted and hybridized to NimbleGen Human DNA microarray. Following this, chip methylation data were read and analyzed. The $\mathrm{CpG}$ island methylation level of sperm was highest (peak value $=15604$ ), followed by oocytes (peak value $=6062$ ). The methylation level of zygotes decreased from 2PN stage (peak value $=3744$ ) to 4-cell stage (peak value $=2826$ ). This methylation level began to rise from 8 -cell stage (peak value $=3073$ ) to morula stage (peak value $=5374)$, ICM stage $($ peak value $=5706)$ and TE stage (peak value $=8376$ ). The proportion of sperm methylation signal that was in the promoter region was $73.7 \%$, and that in the oocyte was $60.8 \%, 2 \mathrm{PN}$ stage was $57.9 \%$, 4-cell stage was $52.2 \%$, 8 -cell stage was $50.3 \%$, morula was $50.3 \%$, ICM was $66.6 \%$ and TE was $66.8 \%$. In conclusion, the current study indicated that $\mathrm{CpG}$ island methylation changes in human preimplantation embryos were divided into three stages. In the first stage from fertilization to $2 \mathrm{PN}$, the level of $\mathrm{CpG}$ island methylation declined sharply. In the second stage from morula
\end{abstract}

Correspondence to: Dr Lei Li, Center for Reproductive Medicine, Third Affiliated Hospital of Guangzhou Medical University, 63 Duobao Road, Guangzhou, Guangdong 510150, P.R. China

E-mail: lileigo@foxmail.com

*Contributed equally

Key words: CpG island methylation, human sperm, human oocyte, preimplantation embryos to blastular ICM, methylation rapidly increased. In the third stage, methylation was reestablished in the TE. Dynamic $\mathrm{CpG}$ island methylation changes were derived primarily from methylation in the promoter region.

\section{Introduction}

DNA methylation serves an essential role in the stability and regulation of gene expression during development and in the maintenance of cellular identity (1). Dynamic changes in DNA methylation are essential to mammalian development as they contribute to cell growth, differentiation, and in particular, early embryonic development $(2,3)$. DNA methylation is associated with critical biological functions, including genomic imprinting, inactivation of the $\mathrm{X}$ chromosome and the regulation of gene expression (4-6). DNA methylation is a chemical modification that typically occurs within a $\mathrm{CpG}$ dinucleotide region in adult somatic cells $(7,8)$. The hypermethylation of promoter $\mathrm{CpG}$ islands affects tumor suppressive mRNAs (9-10). Methylation of promoter $\mathrm{CpG}$ islands present in or near promoter regions may also disrupt the binding of transcription factors (11). Epigenetic modifications, including DNA methylation patterns, represent epigenetic barriers that limit developmental potential during mammalian development (12). Dramatic changes in epigenetic status enable the zygote to erase epigenetic signatures that are inherited from the gametes and subsequently obtain developmental totipotency (12).

Recent advances have begun to elucidate how such dramatic demethylation is activated in the zygote, but a clear picture of the mechanistic details has not yet emerged. Smallwood et al (13) performed the first integrated epigenomic analysis of mammalian oocytes and identified over 1,000 CpG islands that were methylated in mature oocytes. They also demonstrated that methylation of these CpG islands was dependent on DNA methyltransferase $3 \alpha$ and DNA methyltransferase 3-like, but the methylation changes were not distinct at the sequence level. Following fertilization, methylation is comprehensively reprogrammed (9). The maternal genome is demethylated passively and the paternal genome is activated in the zygote during the developmental 
process of the zygote and morula $(9,13)$. Therefore, the establishment of novel methylation landscapes begins. These results provide an important insight into the mechanisms of methylation in germ cells. However, DNA methylome establishment and maintenance in human sperm, oocytes and various developmental stages of the preimplantation embryo have not been described in detail. This is partly due to technical limitations of genome-wide studies in cells, yet this area deserves further exploration (14).

Primordial germ cell differentiation to produce mature gametes, and genome transformation following fertilization are the two important phases of the mammalian life cycle. In previous studies $(15,16)$, quantitative analysis was performed on whole genome methylation sites in human and mammalian gametes and early embryos, which demonstrated the generation of mature gametes in human and mammal fertilized zygotes and DNA methylation and exhibited dynamic changes during preimplantation embryo development. These dynamic patterns of methylation provide an important theoretical basis for understanding gene expression and regulation of the early human embryo, as well as the inhibitory effects of transposons. However, the majority of previous studies used the reduced representation bisulfite sequencing (RRBS) method. Although RRBS can accurately distinguish 5-mC and 5-hmC (10), the coverage rate is low $(10 \% \mathrm{CpG}$ island of the methylation sites) (17). Guo et al (17) used the method of whole genome bisulphite sequencing (WGBS) to detect methylation site changes of the inner cell mass (ICM) and embryo. However, they did not elucidate genome-wide methylation site changes of gametes and the blastula.

The methylated DNA immunoprecipitation (MeDIP) -Chip method can cover almost all promoters and $\mathrm{CpG}$ islands (18). In the present study, MeDIP-Chip was performed to analyze dynamic changes in whole genome $\mathrm{CpG}$ island methylation at various developmental stages of human sperm, oocytes and preimplantation embryos. The current study could improve understanding of the $\mathrm{CpG}$ island and promoter methylation pattern during early embryonic development.

\section{Materials and methods}

Patient information. A total of 43 healthy couples and volunteers (average age, $28.93 \pm 4.15$ years; male: female, 24:19) were recruited at the Third Affiliated Hospital of Guangzhou Medical University (Guangzhou, China) between September 2010 and November 2016. The inclusion criteria were as follows: i) Normal density, vigor and deformity rate in male semen and ii) normal female ovarian function, according to the World Health Organisation criteria for human semen (5th edition) testing standards (19). The exclusion criteria were as follows: i) Age $>40$ years, ii) polycystic ovary syndrome, premature ovarian failure, endometrial polyps or endometriosis, and iii) smoker or alcoholic.

Grouping information. Samples were divided into eight groups based on developmental stage: 1 , Sperm group ( $n=30)$; 2 , MII oocyte group $(\mathrm{n}=25) ; 3$, two-pronuclei $(2 \mathrm{PN})$ period zygote group $(n=25) ; 4$, 4-cell stage embryo group $(n=5) ; 5$, 8-cell stage embryo group $(n=4) ; 6$, morula embryo group $(n=6) ; 7$, blastular ICM group $(\mathrm{n}=5)$; and 8, blastular trophoblastic cells (TE) group $(n=5)$.

Oocyte collection and DNA extraction. Oocytes were collected from patients and volunteers at The Third Affiliated Hospital of Guangzhou Medical University between January 2010 and December 2015.

Under a stereomicroscope, individual eggs $(\sim 140 \mu \mathrm{m}$ in diameter) were collected and placed in Tyrode's solution (SAGE; Cooper Surgical, Trumball, CT, USA). Following the disappearance of the zona pellucida observed under a stereomicroscope, the oocytes ( $0.1 \mathrm{ml} / \mathrm{drop})$ were placed in a buffer containing $2 \mu \mathrm{l}$ lysis buffer A (10 mM Tris-HCl, $1 \mathrm{mM}$ EDTA, $10 \%$ SDS and $1 \%$ proteinase $\mathrm{K} ; \mathrm{pH} 8.0$ ). The cells were placed in a water bath at $37^{\circ} \mathrm{C}$ for $\sim 1 \mathrm{~h}$ to fully lyse the cells. The cells were centrifuged at $11,180 \mathrm{x}$ g for $2 \mathrm{~min}$ at room temperature for $2 \mathrm{~min}$ and stored at $-80^{\circ} \mathrm{C}$ until use. A total of $2 \mu \mathrm{l}$ of the last wash liquid was taken for the polymerase chain reaction (PCR) blank control.

Sperm collection and DNA extraction. The sperm of patients who had accepted in vitro fertilization (IVF) treatment at Guangzhou Medical Third Affiliated Hospital between January 2010 and December 2015 were collected. The patient age ranged between 22 and 40 years. These couples' infertility was not caused by any male factor in semen. Density gradient centrifugation at $400 \mathrm{x} \mathrm{g}$ for $10 \mathrm{~min}$ at $-4^{\circ} \mathrm{C}$ and upstream separation were applied for collection of sperm. The differential lysis method (20) was used, and DNA was extracted using the Genomic DNA Purification Kit (Promega Corporation, Madison, WI, USA) according to the kit protocol, in combination with reagents.

2PN and 4-cell embryo collection and DNA extraction. Intracytoplasmic sperm injection (ICSI) fertilization was performed using oocytes and spermatozoa from the aforementioned sources, and the mature egg was selected. The egg droplet was moved into the field of view. Securing the egg with the holding pipette, the oocyte was transferred to the focal plane under microinjection needle. The polar body was located at the 6-7 or 11-12 o'clock position, so that the injection of the quasi-oocyte was at the 3 o'clock position.

The sperm were pushed to the tip of the injection needle. The injection needle was inserted into the oocyte 3 o'clock position and through the zona pellucida, until the sperm was pushed within the cytoplasm of the middle of the egg. Once the eggs had been injected, they were repeatedly rinsed in a pre-prepared G1.5 Plus Petri dish and placed in incubators containing $5 \% \mathrm{CO}_{2}, 5 \% \mathrm{O}_{2}$ and $90 \% \mathrm{~N}_{2}$ at $37^{\circ} \mathrm{C}$ and cultured in G1.5 Plus droplets separately.

For IVF, GIVF-Plus medium (Vitrolife AB, Göteborg, Sweden) that had been equilibrated overnight was used to prepare fertilized droplets according to the standard of $0.1 \mathrm{ml}$ per microtube droplet per 3 eggs. These fertilized droplets were stored in an incubator $\left(37^{\circ} \mathrm{C}, 6 \% \mathrm{CO}_{2}\right)$ with a covering of mineral oil. A moderate amount of sperm was added to a prepared fertilization dish under a microscope. The concentration was adjusted to $1.0 \times 10^{6} / \mathrm{ml}$. These sperm droplets were stored in an incubator $\left(37^{\circ} \mathrm{C}, 6 \% \mathrm{CO}_{2}\right)$ until fertilization.

Following 3-4 h of pre-incubation, eggs were transferred to the fertilized dish. Two or three eggs were added to one semen 
drop and this fertilized drop was returned to the embryo box for overnight cultivation.

For 2PN collection, prokaryotic cells were observed at 16-20 $\mathrm{h}$ following ICSI or IVF under the inverted microscope and fertilization information was recorded. Two circular structures with nucleolar precursors in the cytoplasm indicated the male and female pronuclei (2PN). Two polar bodies could be observed in the perivitelline space when normal fertilization occurred. DNA was extracted from 12 prokaryotic and ICSI-derived prokaryotes from $2 \mathrm{PN}$-derived IVF using the DNA extraction method as previously described for oocytes.

Two prokaryotic embryos were maintained in culture to D2 at $16-20 \mathrm{~h}$ following ICSI or IVF. These embryos continued to culture until embryos had 4 cells, uniform blastomere size and a low fragmentation rate $(<5 \%)$ was met. A total of 5 embryos were subsequently used in present study. The DNA extraction method was identical to that used for oocytes.

D3 embryo collection and DNA extraction. Following 2 years of successful transplants, donated embryos were stored via the vitrified cryopreservation method. Once thawed, there were 7-9 available embryos, which had uniform size and a low cell debris rate $(<20 \%)$. Five of these embryos were used in the present study. The DNA extraction method was identical to that used for oocytes.

Morula collection and DNA extraction. The thawed D3 embryos were maintained in culture in incubators containing $5 \% \mathrm{CO}_{2}, 5 \% \mathrm{O}_{2}$ and $90 \% \mathrm{~N}_{2}$ at $37^{\circ} \mathrm{C}$. Following overnight culturing, six morula embryos, which were completely fused and with $<20 \%$ fragmentation, were used in the present study. The DNA extraction method was identical to that used for oocytes.

Blastocyst ICM and TE collection and DNA extraction. The aforementioned thawed D3 embryos were cultured to D5 embryos, and high-quality blastocysts were screened. Screening of D5 high-quality blastocysts was based on Garden's grading standards: Blastocysts were grade A or B. Separation of the ICM and TE was performed via mechanical methods; a capillary pipette was used to segregate the ICM and TE under a stereomicroscope. The isolated ICM and TE were washed several times in phosphate-buffered saline and then DNA was extracted using the aforementioned method for oocyte DNA extraction.

MeDIP-Chip. Genomic DNA was extracted using the Wizard ${ }^{\circledR}$ Genomic DNA Purification kit (Promega Corporation), according to the manufacturer's protocol and sonicated to random fragments of 200-1,000 bp. Immunoprecipitation of methylated DNA was performed using Biomag magnetic beads (Polysciences, Inc., Warrington, PA, USA) coupled with a mouse monoclonal 5-methylcytosine antibody (1:100; cat no. C15200081-100; Diagenode, Seraing (Ougrée), Belgium) for $2 \mathrm{~h}$ at $4^{\circ} \mathrm{C}$. DNA was purified by phenol/chloroform extraction and ethanol precipitation. Immunoprecipitated and input DNA were labeled with Cy3- and Cy5-labeled random 9-mers, respectively, and hybridized to a NimbleGen Human DNA Methylation 3x720K CpG Island Plus RefSeq Promoter
Microarray (Roche Diagnostics, Basel, Switzerland). This is a multiplex slide with three identical arrays per slide, and each array includes $27,728 \mathrm{CpG}$ island regions (from approximately $-2,440$ to +610 bp from the transcription start sites) fully covered by $\sim 720,000$ probes. Scanning was performed with the Axon GenePix 4000B microarray scanner (Molecular Devices, LLC, Sunnyvale, CA, USA).

Statistical analysis. Raw data were extracted as paired files by NimbleScan software V2.5 (Roche Diagnostics). Median-centering, quantile normalization and linear smoothing were performed by Bioconductor packages Ringo (www.bioconductor. org/packages/release/bioc/html/Ringo.html), limma (http://www.bioconductor.org/packages/release/bioc/html/ limma.html) and MEDME (www.bioconductor. org/packages/release/bioc/html/MEDME.html). Following normalization, performing median-centering and quantile normalization using Bioconductor packages Ringo and limma generated normalized $\log 2$-ratio data. A modified ACME algorithm (21) was employed where a fixed-length window was slid along the length of each chromosome, testing at each probe using a one-sided Kolmogorov-Smirnov test whether the surrounding window was enriched for high-intensity probes relative to the rest of the array. Each probe had a corresponding P-value score $(-\log 10)$ and a threshold was set to select regions that were enriched in the test sample. From the normalized $\log 2$-ratio data, a sliding-window peak-finding algorithm provided by NimbleScan v2.5 (Roche Diagnostics) was applied to identify the enriched peaks with specified parameters (sliding window width, $750 \mathrm{bp}$; mini probes per peak, 2; P-value minimum cut-off $=2$; maximum spacing between nearby probes within peak, $500 \mathrm{bp}$ ). The identified peaks were mapped to genomic features: Transcripts and CpG islands.

\section{Results}

Dynamic changes in whole-genome CpG island methylation of human preimplantation embryos. $\mathrm{CpG}$ island-associated peak M-value was calculated as a semi-quantitative indicator of the level of $\mathrm{CpG}$ island methylation in human sperm, oocytes and various developmental stages of preimplantation embryo. The peak M-value of sperm was highest $(n=15,604)$, followed by oocytes $(n=6,062)$. The peak M-value of zygotes decreased from $2 \mathrm{PN}$ stage $(n=3,744)$ and reached its minimum level at the 4-cell stage $(n=2,826)$. This peak M-value began to rise from 8 -cell stage $(n=3,073)$ to morula stage $(n=5,374)$ and to ICM stage $(n=5,706)$ and TE stage $(n=8,376$; Fig. 1$)$. The peak M-value of the ICM stage was similar to that of oocytes. The peak M-value of TE fell between those of oocytes and sperm.

Dynamic changes in the whole-genome $\mathrm{CpG}$ island methylation pattern of human preimplantation embryos were characterized by low (peak M-value $\leq 0.4$ ) and high (peak $\mathrm{M}$-value $\geq 0.7$ ) $\mathrm{CpG}$ island methylation regions. The proportion of high CpG island methylation regions (peak M-value $\geq 0.7$ ) in $2 \mathrm{PN}$ stage was lowest. After the $2 \mathrm{PN}$ stage, $\mathrm{CpG}$ island methylation tended to increase. The proportion of high $\mathrm{CpG}$ island methylation regions in the blastular ICM stage was similar to those in the TE stage. However, the results for the proportion 
Table I. Proportion of low, middle and high CpG island-associated peak M-values.

\begin{tabular}{lcccccccr}
\hline & \multicolumn{8}{c}{ Stage $(\%)$} \\
\cline { 2 - 9 } Peak M-value & Sperm & Oocyte & 2PN & 4-cell & 8-cell & Morula & ICM & TE \\
\hline Low & 13.90 & 28.84 & 32.05 & 30.33 & 32.18 & 26.57 & 28.58 & 25.76 \\
Middle & 34.77 & 36.89 & 37.39 & 36.41 & 36.45 & 36.30 & 35.96 & 38.44 \\
High & 51.33 & 34.28 & 30.56 & 33.26 & 31.37 & 37.12 & 35.45 & 35.79
\end{tabular}

ICM, inner cell mass; TE, trophoblast cells; 2PN, two pronuclei.

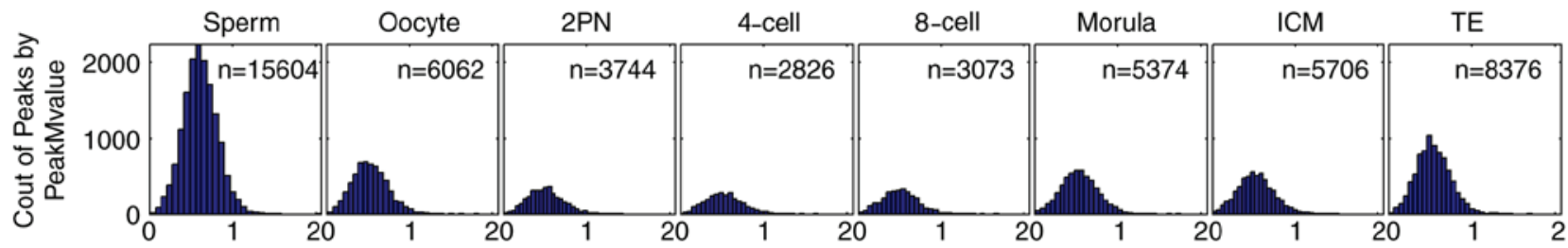

Figure 1. $\mathrm{CpG}$ island-associated peak M-value in sperm $(\mathrm{n}=15,604)$, oocyte $(\mathrm{n}=6,062), 2 \mathrm{PN}$ stage $(\mathrm{n}=3,744)$, 4-cell stage $(\mathrm{n}=2,826), 8$-cell stage $(\mathrm{n}=3,073)$, morula stage $(\mathrm{n}=5,374)$, ICM $(\mathrm{n}=5,706)$ and TE $(\mathrm{n}=8,376)$. Horizontal axis: $\mathrm{CpG}$-island associated peak M-value. The peak M-value of sperm was highest $(n=15,604)$, followed by oocytes $(n=6,062)$. The peak M-value of zygotes decreased from 2PN stage $(n=3,744)$ and reached its minimum level at the 4-cell stage $(n=2,826)$. This peak M-value began to rise from 8-cell stage $(n=3,073)$ to morula stage $(n=5,374)$ and to ICM stage $(n=5,706)$ and TE stage $(n=8,376)$. The peak M-value of the ICM stage was similar to that of oocytes. The peak M-value of TE fell between those of oocytes and sperm. ICM, inner cell mass; $\mathrm{TE}$, trophoblast cells; 2PN, two pronuclei.

of low $\mathrm{CpG}$ island methylation regions (peak M-value $\leq 0.4$ ) were reversed. The proportion of low $\mathrm{CpG}$ island methylation regions (peak M-value $\leq 0.4$ ) in $2 \mathrm{PN}$ stage was highest. Changes in the proportion of middle $\mathrm{CpG}$ island methylation regions were not evident (Table I and Fig. 2).

Variations in methylation level of whole genome CpG islands of preimplantation embryos. In the first stage from fertilization to $2 \mathrm{PN}$, the level of $\mathrm{CpG}$ island methylation declined sharply. In the second stage from morula to blastular ICM, methylation rapidly increased again. The third stage was the methylation reestablishment process of TE (Table II and Fig. 3).

Dynamic changes in CpG island methylation patterns in intragenic, intergenic and promoter regions. The $\mathrm{CpG}$ island methylation levels of zygotes decreased from the 2PN stage and reached a minimum level at the 4 -cell stage. The methylation level began to rise from the 8-cell stage to the ICM stage, reaching a similar level to the oocyte. These dynamic changes were derived from methylation in the promoter region. The proportion of sperm methylation signal in the promoter region was $73.7 \%$, and that in the oocyte was $60.8 \%, 2 \mathrm{PN}$ was $57.9 \%$, 4-cell stage was $52.2 \%$, 8-cell stage was $50.3 \%$, morula stage was $68.8 \%$, ICM was $66.6 \%$ and TE was $66.8 \%$. Methylation fluctuation in the intergenic region was less obvious than those in promoter region. However, significant fluctuation of dynamic methylation changes in intragenic regions were not observed (Fig. 4).

The mean values of $\mathrm{CpG}$ island-associated PeakScore indicated that dynamic demethylation changes in intragenic, intergenic and promoter regions were all observed in the transformation process between sperm, oocyte and zygote (Table III and Fig. 5).
Fluctuation at various stages of the preimplantation embryo was primarily evident in the promoter region. In promoter regions, the methylation level reached a minimum value in the 2PN stage, and subsequently began to rise. The methylation level of ICM and TE in the promoter region fell between the levels observed in oocytes and sperm. The $\mathrm{CpG}$ methylation fluctuation pattern in intragenic regions was similar to those in intergenic regions. In intragenic and intergenic regions, the methylation level decreased from 2PN stage and reached a minimum value at the 4-cell stage, and subsequently began to rise. The methylation level of ICM and TE in these regions was similar to that in oocytes (Fig. 5).

\section{Discussion}

DNA methylation, an enzymatic modification at the 5'position of a cytosine pyrimidine ring, is of great importance in cellular processes, including genome development and regulation (22). This modification may recruit methyl-CpG binding proteins to act as a 'silencing' epigenetic mark (23). Changes in DNA methylation patterns are important in investigating the roles of epigenetics in the pathogenesis of diseases, and the patterns are subject to complex regulation during reprogramming $(24,25)$. Advances in the field of DNA methylation have been made due to the development of sequencing technology. The majority of previous studies have either focused on global DNA methylation, low resolution or candidate gene DNA methylation changes using sequencing methods, such as bisulfite pyrosequencing (26) and RRBS (27). RRBS is one of several sequencing methods applied to profile DNA methylation (27). Although RRBS can accurately distinguish 5-mC and 5-hmC, it only covers $10 \%$ of all $\mathrm{CpG}$ sites, which may leave $\mathrm{CpG}$-sparse regions unexplored in the human 


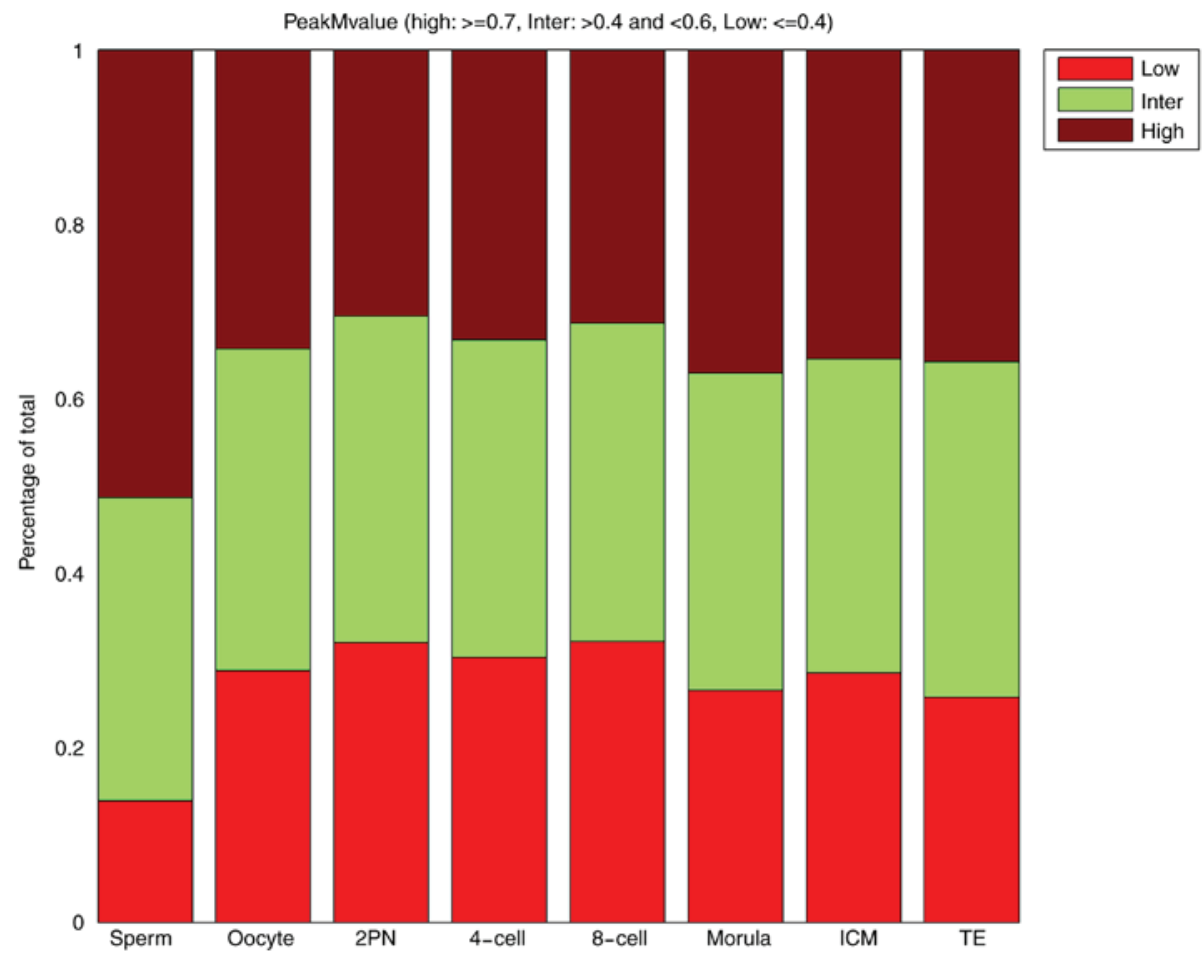

Figure 2. Proportion of low, middle and high $\mathrm{CpG}$ island-associated peak $\mathrm{M}$-values. $\mathrm{CpG}$ island methylation changes of human preimplantation embryos are divided into three stages. The proportion of high $\mathrm{CpG}$ island methylation regions (peak M-value $\geq 0.7$ ) in $2 \mathrm{PN}$ stage was lowest. Following the $2 \mathrm{PN}$ stage, fluctuations in $\mathrm{CpG}$ island methylation tended to increase. The proportion of high $\mathrm{CpG}$ island methylation regions in the blastular ICM stage was similar to those in the TE stage. However, the results for the proportion of low $\mathrm{CpG}$ island methylation regions (peak M-value $\leq 0.4$ ) were reversed. The proportion of low $\mathrm{CpG}$ island methylation regions (peak M-value $\leq 0.4$ ) in $2 \mathrm{PN}$ stage was highest. Changes in the proportion of high $\mathrm{CpG}$ island methylation regions were not evident. ICM, inner cell mass; TE, trophoblast cells; 2PN, two pronuclei.

genome (15). To improve coverage rate and obtain absolute quantification of DNA methylation, Guo et al (15) performed WGBS on the blastular ICM and post-implantation embryos. However, this sequencing method requires a higher DNA input. The study did not elucidate genome-wide methylation site changes of gametes and blastular embryos, which are difficult to collect. Post-Bisulfite Adaptor Tagging (PBAT) was also applied to profile DNA methylation (28). The PBAT method could generate a substantial number of unamplified reads from as little as subnanogram quantities of DNA (29). However, site preferences in the random priming steps would lead to 'pile-ups' of reads (29). Differential priming between methylated and unmethylated alleles may lead to inaccurate estimation of methylation level (29).

MeDIP sequencing is a popular $5 \mathrm{mC}$ capture-based method, which can detect genome-wide DNA methylation levels rapidly and cost-efficiently at a resolution of $100-500 \mathrm{bp}$ (30). This sequencing method requires low-input DNA and has broad application prospects in the study of DNA methylation (24). In the present study, MeDIP-Chip was performed to investigate dynamic changes in whole genome $\mathrm{CpG}$ island methylation in human sperm, oocytes and various developmental stages of the preimplantation embryo.

The majority of array-based studies are performed based on immunoprecipitation of methylated DNA, coupled with hybridization to MeDIP-chip $(27,31)$. MeDIP-Chip may be a sufficient tool to detect differentially methylated regions at the level of several hundred base pairs rather than at the level of single cytosines (32). Among several DNA methylation analysis methods, MeDIP-chip was previously reported to be a suitable method to detect methylated DNA information when taking into account cost, ease of implementation and sensitivity $(33,34)$. This technology performs well in detecting DNA methylation information at probe-level resolution, with a low genome-wide combined false-positive and false-negative rate of approximately 0.21 (32). However, detection of DNA methylation information can be susceptible to strong signal distortions, which result from dye bias and the CG content of effectively unmethylated genomic regions (32).

The current results demonstrated that dynamic changes in the pattern of $\mathrm{CpG}$ island methylation were present in human sperm, oocytes and various developmental stages of the preimplantation embryo. The level of $\mathrm{CpG}$ island methylation in human sperm was highest among all the experimental groups. Demethylation in the zygote began from the pronucleus stage and methylation reached a minimum level at the 4-cell stage following fertilization. Methylation was then increased until it was reestablished at the blastular ICM stage. The level of $\mathrm{CpG}$ island methylation in TE was between that of oocytes and sperm. This pattern of methylation change was consistent with previous studies: Methylation in human or mammalian gametes and early embryos was previously identified to change over different developmental stages, but there are differences in the pattern of changes between species $(15,35,36)$. In genome-scale maps of DNA methylation in gametes and over the preimplantation timeline, demethylation of the preimplantation embryo in mice began from the pronuclear stage. A gradual decrease in methylation was observed from the zygote 
Table II. Gene CpG island methylation changes from gametes to various developmental stages of the preimplantation embryo.

\begin{tabular}{lccccccc}
\hline Trend & Sp to 2PN & Oo to 2PN & $\begin{array}{c}\text { 2PN to } \\
\text { 4-cell }\end{array}$ & $\begin{array}{c}\text { 4-cell to } \\
\text { 8-cell }\end{array}$ & $\begin{array}{c}\text { 8-cell } \\
\text { to morula }\end{array}$ & $\begin{array}{c}\text { Morula to } \\
\text { ICM }\end{array}$ & ICM to TE \\
\hline Stable (n) & 14,397 & 6,277 & 4,336 & 3,965 & 6,401 & 6,096 & 8,086 \\
Changing (n) & 2,102 & 1,656 & 1,070 & 931 & 923 & 2,330 & 2,776 \\
\hline
\end{tabular}

$\mathrm{n}$, stable or changing peak value; Sp, sperm; Oo, oocytes; ICM, inner cell mass; TE, trophoblast cells; 2PN, two pronuclei.

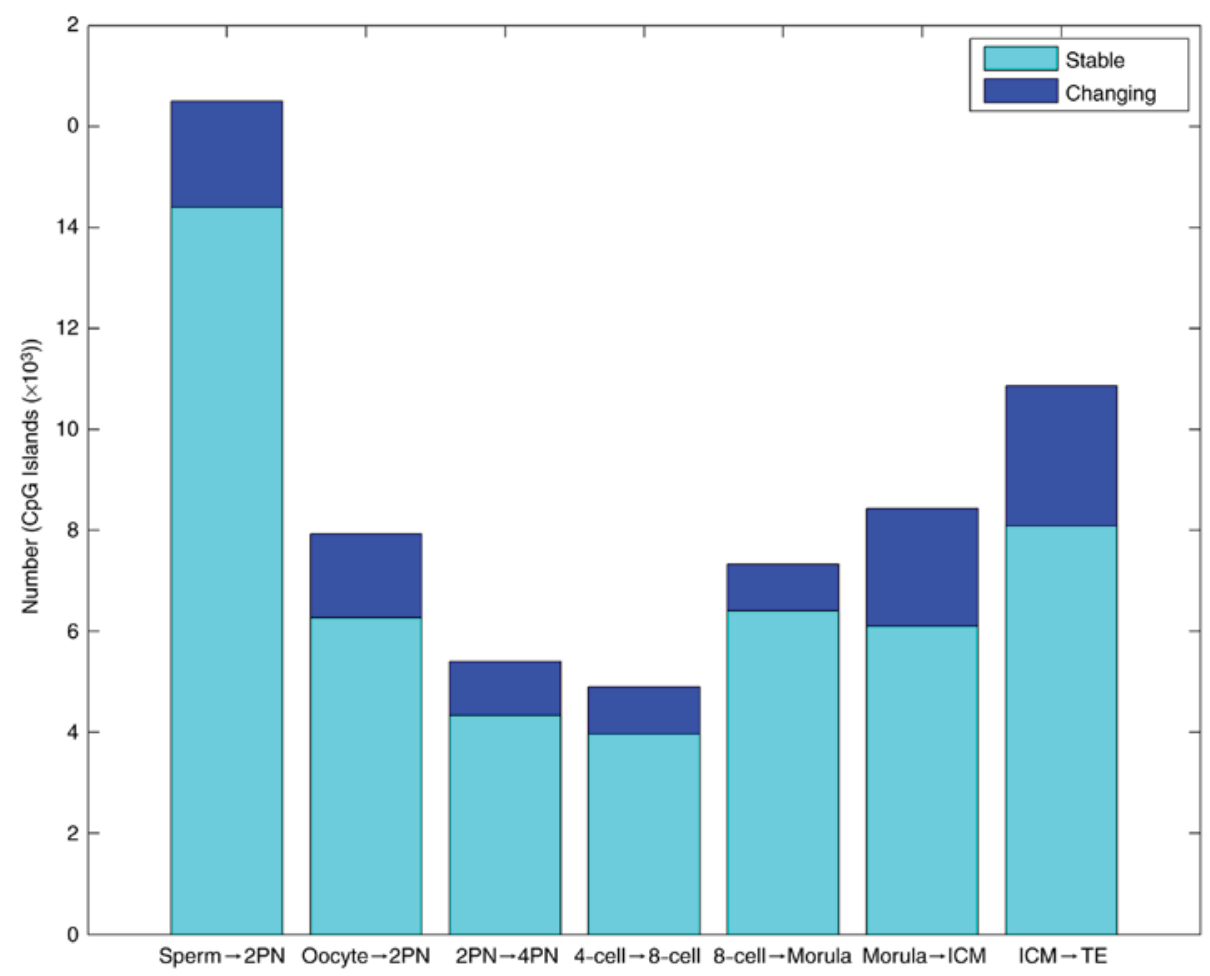

Figure 3. CpG island methylation changes from gametes to various developmental stages of the preimplantation embryo. Dark blue represents the peak value of the changing methylation point, and light blue represents the peak value of the stable methylation point. In the first stage from fertilization to 2PN, the level of $\mathrm{CpG}$ island methylation declined sharply. In the second stage from morula to blastular ICM, methylation rapidly increased again. The third stage was the methylation reestablishment process of TE. ICM, inner cell mass; TE, trophoblast cells; 2PN, two pronuclei.

through the pronuclear stage and into the blastular ICM, where methylation levels reached a minimum (35).

Methylation patterns are similar between mouse and human embryos. In genome-scale maps of DNA methylation in the human preimplantation embryo, methylation levels of the blastular ICM exhibited the lowest values (15). However, genome-wide demethylation in mouse embryos predominantly occurs at the 1-cell stage, while demethylation in the human embryo occurs from fertilization to the 2-cell stage (15). In the present study, the lowest methylation level occurred at the 4-cell stage. This result indicated that the change in $\mathrm{CpG}$ island methylation was primarily due to $\mathrm{CpG}$ island methylation fluctuation in promoter regions, suggesting that $\mathrm{CpG}$ island methylation of the 4-cell stage primarily occurs in promoter regions. Methylation levels in promoter regions have been demonstrated to be associated with the transcriptional activity of genes (37). Therefore, $\mathrm{CpG}$ island methylation at the 4-cell stage may serve an important role in the conversion of maternal genes to zygotic genes. Various
DNA regions of oocytes are in a demethylated state $(38,39)$. The methylation status of oocytes is a powerful predictor of zygotic methylation level and is thought to dictate the zygotic methylation landscape (35). Hypomethylated regions in the oocyte could indicate disparities between the sperm and early embryo (35).

Sperm contribute to the methylation patterns of the zygote by altering the methylation level of some specific retroelement subfamilies (35). Disparities between sperm and oocytes result in different expression patterns due to epigenetics. Mammalian sperm present with a high DNA methylation level $(35,36)$. However, the DNA methylation levels of mammalian and human oocytes are lower compared with those in sperm $(35,36)$. Following fertilization, sperm and oocyte DNA in the zygote undergoes a series of changes. Erasure of DNA methylation may be an important mechanism in early embryo development. The results of the current study demonstrated that the DNA CpG island methylation pattern in the blastular ICM, the final stage before embryo implantation, is similar to 


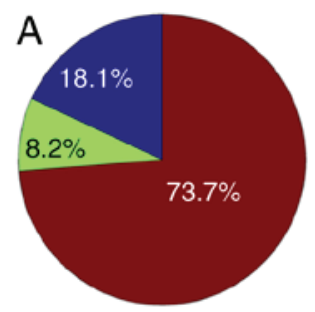

Sperm

E

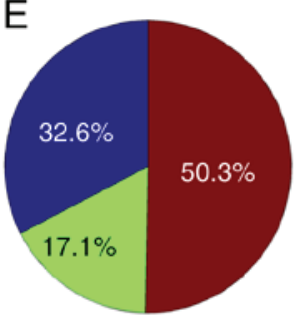

8-cell

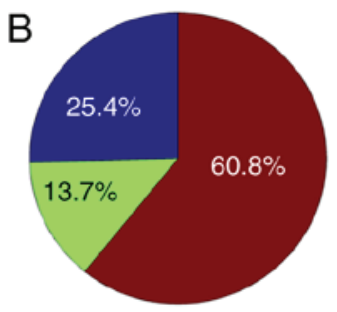

Oocyte

$\mathrm{F}$

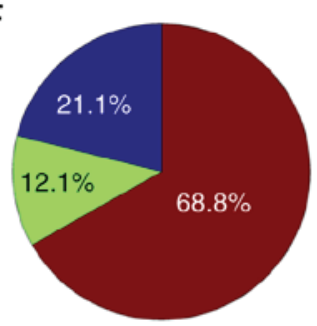

Morula

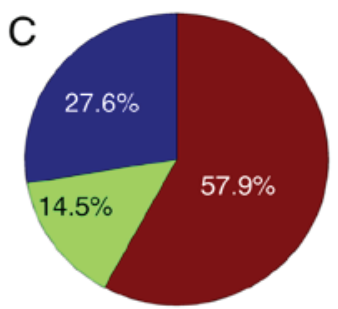

2PN

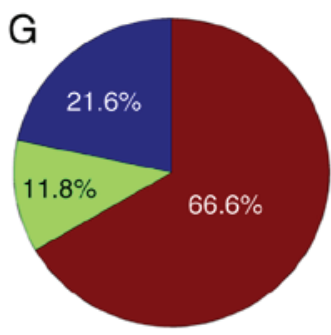

ICM

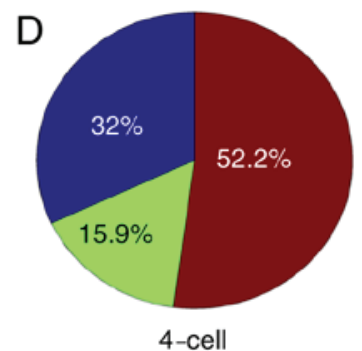

4-cell

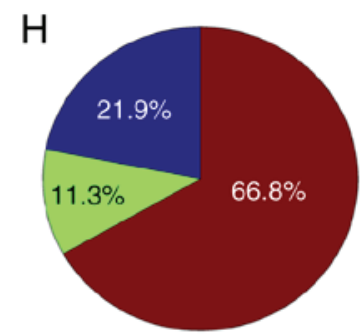

TE

\begin{tabular}{l|l|l} 
Intragenic & Intergenic \\
\hline
\end{tabular}

Figure 4. CpG island-associated PeakScore in intragenic, intergenic and promoter regions. (A) Sperm, (B) oocyte, (C) 2PN stage, (D) 4-cell stage, (E) 8-cell stage, (F) morula stage, (G) ICM and (H) TE. The proportion of sperm methylation signal in the promoter region was $73.7 \%$, and that in the oocyte was $60.8 \%$, $2 \mathrm{PN}$ was $57.9 \%$, 4-cell stage was $52.2 \%, 8$-cell stage was $50.3 \%$, morula stage was $68.8 \%$, ICM was $66.6 \%$ and TE was $66.8 \%$. Methylation in the intergenic region took second place. However, dynamic methylation changes in intragenic regions were not observed. ICM, inner cell mass; TE, trophoblast cells; 2PN, two pronuclei.
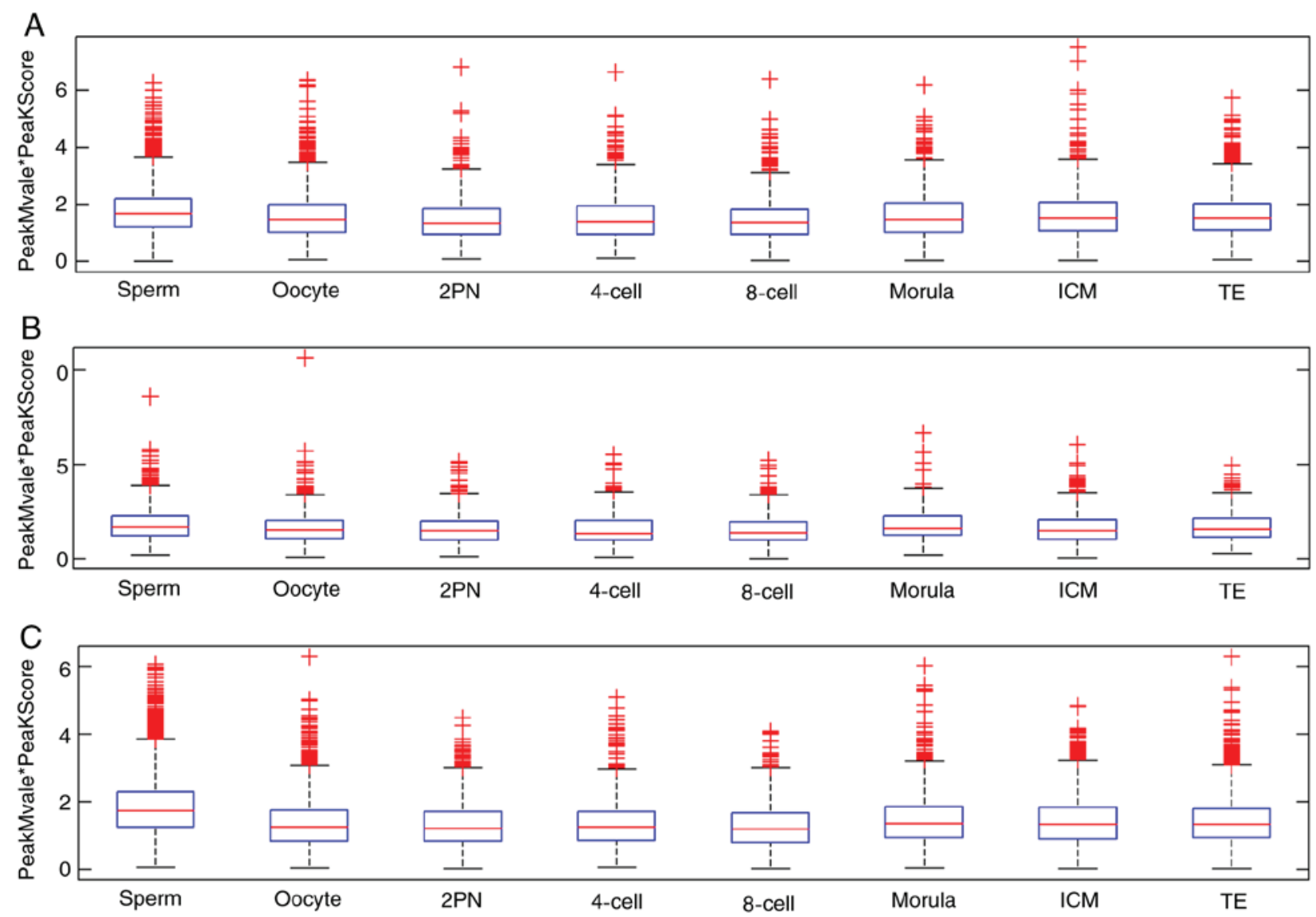

Figure 5. Mean values of $\mathrm{CpG}$ island-associated peak M-value PeakScore in (A) intergenic, (B) intragenic and (C) promoter regions. Fluctuation at various stages of the preimplantation embryo was most evident in the promoter region. In promoter regions, the methylation level reached a minimum value in the $2 \mathrm{PN}$ stage, then it began to rise. The methylation level of ICM and TE in the promoter region fell between the levels observed in oocytes and sperm. The CpG methylation fluctuation pattern in intragenic regions was similar to those in intergenic regions. In intragenic and intergenic regions, the methylation level decreased from $2 \mathrm{PN}$ stage and reached a minimum value at the 4-cell stage, then it began to rise. The methylation level of ICM and TE in these regions was similar to that in oocytes. ICM, inner cell mass; TE, trophoblast cells; $2 \mathrm{PN}$, two pronuclei. 
that in oocytes. Compared with sperm, the $\mathrm{CpG}$ island methylation pattern in oocytes is more significant.

The current results provide insight into the dynamic changes in whole genome $\mathrm{CpG}$ island methylation in human sperm, oocytes and various developmental stages of preimplantation embryo. The results indicated that the level of $\mathrm{CpG}$ island methylation in human sperm was higher compared with oocytes and various developmental stages of the preimplantation embryo. Demethylation in the zygote began from the pronucleus stage and methylation reached a minimum level at the 4-cell stage following fertilization. Methylation then increased until it was reestablished at the blastular ICM stage, at which point the methylation level was similar to that in oocytes. The level of $\mathrm{CpG}$ island methylation in TE was between that of oocytes and sperm. The global methylation level of the preimplantation embryo reached its minimum value at the ICM stage. It was noted that $\mathrm{CpG}$ methylation erasure of the preimplantation embryo primarily appeared during the 4-cell stage, and whole genome erasure primarily appeared in the 2-cell stage. It was also demonstrated that dynamic changes in $\mathrm{CpG}$ methylation were derived from methylation of promoter regions. The current MeDIP-Chip analysis results provide an insight into the dynamic methylation patterns of whole genome $\mathrm{CpG}$ islands and methylation in human sperm, oocytes and embryos.

In conclusion, the current study suggested that $\mathrm{CpG}$ island methylation changes in human preimplantation embryos were divided into three stages. In the first stage from fertilization to 2PN, the level of $\mathrm{CpG}$ island methylation declined sharply. In the second stage from the morula to the blastular ICM, methylation rapidly increased again. In the third stage, methylation was reestablished in the TE. Dynamic $\mathrm{CpG}$ island methylation changes were primarily derived from methylation in promoter regions; however further validation experiments are required to examine the methylation variability in larger cohorts. The current study therefore provides a basis for further epigenetic studies focused on early zygote development.

\section{Acknowledgements}

Not applicable.

\section{Funding}

The present study was supported by grants from the Guangdong Natural Science Youth Fund (grant no 2018A030310167), Guangdong Provincial Department of Education Featured Innovation Project (grant no. 2017KTSCX153), Guangzhou Liwan District Science and Technology Plan Project (grant no. 201704037), the National Nature Science Foundation of China (grant no. 81502507), the National Nature Science Foundation of China (grant no. 81871211) and the National Nature Science Foundation of China (grant no. 81801532).

\section{Availability of data and materials}

The microarray data (GSE124358) is freely available from the GEO datasets of the NCBI website (www.ncbi.nlm.nih. gov/geo/query/acc.cgi?acc=GSE124358). Analysis of these microarray data for this study will be made available from the corresponding author on reasonable request.

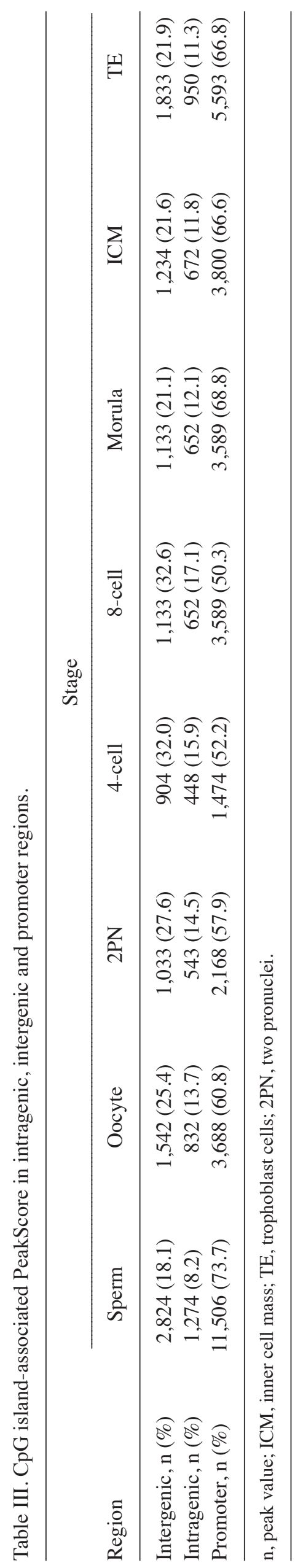




\section{Authors' contributions}

LL and YH contributed to the conception and coordination of the study. YH and HL designed the study and prepared the manuscript. YH, HL, HD, WZ and XK analyzed the data and interpreted the results. YL and XZ collected and processed the samples. All authors read and approved the final manuscript.

\section{Ethics approval and consent to participate}

The present study was approved by the Reproductive Study Ethics Committee of the Third Affiliated Hospital of Guangzhou Medical University (Guangzhou, China). All sperm, embryos were collected from donor couples that provided written informed consent. Oocyte samples were collected from donor couples or volunteers that provided their written informed consent.

\section{Patient consent for publication}

All patients or volunteers recruited provided written informed consent for publication.

\section{Competing interests}

The authors declare that they have no competing interests.

\section{References}

1. Stelzer Y, Shivalila CS, Soldner F, Markoulaki S and Jaenisch R: Tracing dynamic changes of DNA methylation at single-cell resolution. Cell 163: 218-229, 2015.

2. Smith ZD and Meissner A: DNA methylation: Roles in mammalian development. Nat Rev Genet 14: 204-220, 2013.

3. He W, Kang X, Du H, Song B, Lu Z, Huang Y, Wang D, Sun X, $\mathrm{Yu}$ Y and Fan Y: Defining differentially methylated regions specific for the acquisition of pluripotency and maintenance in human pluripotent stem cells via microarray. PLoS One 9: e108350, 2014.

4. Jaenisch R and Bird A: Epigenetic regulation of gene expression: How the genome integrates intrinsic and environmental signals. Nat Genet (33 Suppl): S245-S254, 2003.

5. Dhiman VK, Attwood K, Campbell MJ and Smiraglia DJ: Hormone stimulation of androgen receptor mediates dynamic changes in DNA methylation patterns at regulatory elements. Oncotarget 6: 42575-42589, 2015.

6. Ping W, Hu J, Hu G, Song Y, Xia Q, Yao M, Gong S, Jiang C and Yao H: Genome-wide DNA methylation analysis reveals that mouse chemical iPSCs have closer epigenetic features to mESCs than OSKM-integrated iPSCs. Cell Death and Disease 9: 187, 2018.

7. Baylin SB: DNA methylation and gene silencing in cancer. Nat Clin Pract Oncol 1 (2 Suppl): S4-S11, 2005.

8. Li HJ, Wan RP, Tang LJ, Liu SJ, Zhao QH, Gao MM, Yi YH, Liao WP, Sun XF and Long YS: Alteration of Scn3a expression is mediated via $\mathrm{CpG}$ methylation and MBD2 in mouse hippocampus during postnatal development and seizure condition. Biochim Biophys Acta 1849: 1-9, 2015.

9. Morgan HD, Santos F, Green K, Dean W and Reik W: Epigenetic reprogramming in mammals. Hum Mol Genet 14: R47-R58, 2005.

10. Gu Y, Zhang Z, Yin J, Ye J, Song Y, Liu H, Xiong Y, Lu M, Zheng $\mathrm{G}$ and $\mathrm{He} \mathrm{Z}$ : Epigenetic silencing of miR-493 increases the resistance to cisplatin in lung cancer by targeting tongue cancer resistance-related protein 1 (TCRP1). J Exp Clin Cancer Res 36: 114, 2017.

11. Dexheimer GM, Alves J, Reckziegel L, Lazzaretti G and Abujamra AL: DNA methylation events as markers for diagnosis and management of acute myeloid leukemia and myelodysplastic syndrome. Dis Markers 2017: 5472893, 2017.
12. Seisenberger S, Peat JR, Hore TA, Santos F, Dean W and Reik W: Reprogramming DNA methylation in the mammalian life cycle: Building and breaking epigenetic barriers. Philos Trans R Soc Lond B Biol Sci 368: 20110330, 2013.

13. Smallwood SA, Tomizawa S, Krueger F, Ruf N, Carli N, Segonds-Pichon A, Sato S, Hata K, Andrews SR and Kelsey G: Dynamic $\mathrm{CpG}$ island methylation landscape in oocytes and preimplantation embryos. Nat Genet 43: 811-814, 2011.

14. Yu B, Russanova VR, Gravina S, Hartley S, Mullikin JC, Ignezweski A, Graham J, Segars JH, DeCherney AH and Howard BH: DNA methylome and transcriptome sequencing in human ovarian granulosa cells links age-related changes in gene expression to gene body methylation and 3'-end GC density. Oncotarget 6: 3627-3643, 2015.

15. Guo H, Zhu P, Yan L, Li R, Hu B, Lian Y, Yan J, Ren X, Lin S, Li J, et al: The DNA methylation landscape of human early embryos. Nature 511: 606-610, 2014.

16. Jiang Z, Lin J, Dong H, Zheng X, Marjani SL, Duan J, Ouyang Z, Chen J and Tian XC: DNA methylomes of bovine gametes and in vivo produced preimplantation embryos. Biol Reprod 99: 949-959, 2018.

17. Guo H, Zhu P, Yan L, Li R, Hu B, Lian Y, Yan J, Ren X, Lin S, Li J, et al: The DNA methylation landscape of human early embryos. Nature 511: 606-610, 2014.

18. Down TA, Rakyan VK, Turner DJ, Flicek P, Li H, Kulesha E, Gräf S, Johnson N, Herrero J, Tomazou EM, et al: A Bayesian deconvolution strategy for immunoprecipitation-based DNA methylome analysis. Nat Biotech 26: 779-785, 2008.

19. Cao XW, Lin K, Li CY and Yuan CW: A review of WHO Laboratory Manual for the Examination and Processing of Human Semen (5th edition). Zhonghua Nan Ke Xue 17: 1059-1063, 2011 (In Chinese).

20. Yoshida K, Sekiguchi K, Mizuno N, Kasai K, Sakai I, Sato H and Seta S: The modified method of two-step differential extraction of sperm and vaginal epithelial cell DNA from vaginal fluid mixed with semen. Forensic Sci Int 72: 25-33, 1995.

21. Scacheri PC, Crawford GE and Davis S. Statistics for ChIP-chip and DNase hypersensitivity experiments on NimbleGen arrays. Methods Enzymol 411: 270-282, 2006.

22. Holliday R and Pugh JE: DNA modification mechanisms and gene activity during development. Science 187: 226-232, 1975.

23. Jones PA: Functions of DNA methylation: Islands, start sites, gene bodies and beyond. Nat Rev Genet 13: 484-492, 2012.

24. Xiao Y, Yu F, Pang L, Zhao H, Liu L, Zhang G, Liu T, Zhang H, Fan H, Zhang Y, et al: MeSiC: A model-based method for estimating $5 \mathrm{mC}$ levels at single-CpG resolution from MeDIP-seq. Sci Rep 5: 14699, 2015

25. He S, Sun H, Lin L, Zhang Y, Chen J, Liang L, Li Y, Zhang M, Yang X, Wang X, et al: Passive DNA demethylation preferentially up-regulates pluripotency-related genes and facilitates the generation of induced pluripotent stem cells J Biol Chem 292: 18542-18555, 2017.

26. Vinci G, Buffat C, Simoncini S, Boubred F, Ligi I, Dumont F, Le Bonniec B, Fournier T, Vaiman D, Dignat-George F and Simeoni U: Gestational age-related patterns of AMOT methylation are revealed in preterm infant endothelial progenitors. PLoS One 12: e0186321, 2017.

27. Beck S and Rakyan VK: The methylome: Approaches for global DNA methylation profiling. Trends Genet 24: 231-237, 2008.

28. Zhu P, Guo H, Ren Y, Hou Y, Dong J, Li R, Lian Y, Fan X, Hu B, Gao Y, et al: Single-cell DNA methylome sequencing of human preimplantation embryos. Nat Genet 50: 12-19, 2018.

29. Miura F, Enomoto Y, Dairiki R and Ito T: Amplification-free whole-genome bisulfite sequencing by post-bisulfite adaptor tagging. Nucleic Acids Res 40: e136, 2012.

30. Taiwo O, Wilson GA, Morris T, Seisenberger S, Reik W, Pearce D, Beck S and Butcher LM: Methylome analysis using MeDIP-seq with low DNA concentrations. Nat Protoc 7: 617-636, 2012.

31. Harrison A and Parle-McDermott A: DNA methylation: A timeline of methods and applications. Front Genet 2: 74, 2011.

32. Wardenaar R, Liu H, Colot V, Colomé-Tatché M and Johannes F: Evaluation of MeDIP-chip in the context of whole-genome bisulfite sequencing (WGBS-seq) in Arabidopsis. Methods Mol Biol 1067: 203-224, 2013

33. Cortijo S, Wardenaar R, Colomé-Tatché M, Johannes F and Colot V: Genome-wide analysis of DNA methylation in Arabidopsis using MeDIP-chip. Methods Mol Biol 1112: 125-149, 2014. 
34. Seifert M, Cortijo S, Colomé-Tatché M, Johannes F, Roudier F and Colot V: MeDIP-HMM: Genome-wide identification of distinct DNA methylation states from high-density tiling arrays. Bioinformatics 28: 2930-2939, 2012.

35. Smith ZD, Chan MM, Mikkelsen TS, Gu H, Gnirke A, Regev A and Meissner A: A unique regulatory phase of DNA methylation in the early mammalian embryo. Nature 484: 339-344, 2012

36. Smith ZD, Chan MM, Humm KC, Karnik R, Mekhoubad S, Regev A, Eggan K and Meissner A: DNA methylation dynamics of the human preimplantation embryo. Nature 511: 611-615, 2014

37. Klose RJ and Bird AP: Genomic DNA methylation: The mark and its mediators. Trends Biochem Sci 31: 89-97, 2006.
38. Guo F, Li X, Liang D, Li T, Zhu P, Guo H, Wu X, Wen L, Gu TP, $\mathrm{Hu} \mathrm{B}$, et al: Active and passive demethylation of male and female pronuclear DNA in the mammalian zygote. Cell Stem Cell 15: 447-459, 2014

39. Hajkova P, Jeffries SJ, Lee C, Miller N, Jackson SP and Surani MA: Genome-wide eprogramming in the mouse germ line entails the base excision repair pathway. Science 329: 78-82, 2010.

(i) (i) $(9)$ This work is licensed under a Creative Commons Attribution-NonCommercial-NoDerivatives 4.0 International (CC BY-NC-ND 4.0) License. 